Didáctica. Lengua y literatura

ISSN: 1130-0531

http://dx.doi.org/10.5209/DIDA.61960

\title{
Multiculturalidad en el aula de segundas lenguas: la literatura como recurso en Educación Primaria
}

\author{
Cristina Molino Fernández'; Laura Mier Pérez²
}

Recibido: 24 de mayo de 2017 / Aceptado: 24 de julio de 2018

Resumen. En este artículo presentamos una investigación que versa sobre la importancia de la literatura de contextos culturales múltiples en el aula de segundas lenguas. En concreto, la revisión bibliográfica está encaminada a apoyar la propuesta de algunas lecturas que sirvan como ejemplo de cómo llevar a cabo en el aula de Educación Primaria la lectura de textos literarios que sustenten la competencia cultural y comunicativa del discente de inglés como lengua extranjera en Educación Primaria.

Palabras clave: Literatura; multiculturalidad; competencia comunicativa; competencia cultural.

\section{[en] Multiculturalism in Second Language Classrooms: literature as a resource in Primary Education}

Abstract. In this paper we present research about the importance of literature from multiple cultural backgrounds in the second language classroom. The literature review supports a proposal for specific readings. These are used as an example on how to incorporate texts in the classroom that motivate the development of cultural and communicative competences for students of English as a second language in a primary education context.

Keywords: Literature; cultural diversity; communicative competence; cultural competence.

\section{[fr] Multiculturalité dans les secondes langues: la littérature comme une ressource en Éducation primaire}

Résumé. Cet article présente un travail de recherche portant sur l'importance d'introduire la littérature provenant de contextes multiculturels dans le cadre de l'enseignement de langues étrangères. Plus particulièrement, cette révision bibliographique a pour but de d'appuyer l'introduction d'un certain nombre de lectures qui permettraient d'illustrer comment mener à bien la lecture de textes littéraires à partir desquels développer la compétence culturelle et communicative de l'apprenant dans le cadre de l'enseignement de l'anglais en Éducation primaire.

Mots-clés: Littérature; multiculturalité; compétence communicative; compétence culturelle.

Sumario. 1. Introducción. 2. Planteamiento teórico-descriptivo. 3. La literatura infantil en el aula de segundas lenguas. Situación. 4. Propuesta de mejora: dos lecturas. 5. Conclusiones. 6. Referencias bibliográficas.

\footnotetext{
1 Departamento de Filología. Universidad de Cantabria (España)

E-mail: cristimf93@gmail.com

2 Departamento de Filología. Universidad de Cantabria (España)

E-mail: laura.mier@unican.es
} 
Cómo citar: Molino Fernández, C.; Mier Pérez, L. (2018) Multiculturalidad en el aula de segundas lenguas: la literatura como recurso en Educación Primaria, Didáctica. Lengua y Literatura, 30, 157-168.

\section{Introducción}

Dadas las coordenadas educativas en las que nos movemos actualmente es cada vez más necesario adaptar los contenidos de las aulas a nuestra sociedad cambiante. De ahí que consideremos necesaria la sistemática revisión de las lecturas y de los materiales que proponemos a nuestros alumnos. En concreto, en este caso realizamos una reflexión sobre la situación metodológica de la enseñanza de segundas lenguas y de la relación que esta mantiene con la literatura para concluir la importancia de su presencia como parte integral del desarrollo de la competencia comunicativa. Más aún, la configuración de nuestra sociedad dentro del mundo globalizado llama a la consideración cada vez mayor de lecturas que reflejen la riqueza cultural de nuestro entorno cercano y lejano, por ello, nuestra propuesta, ofrece modelos de lectura cuyo contexto cultural está alejado del discente para el que están pensados. De especial importancia nos parece, además, la incidencia en los primeros años de la vida educativa, tanto por la progresiva implantación del inglés a edades cada vez más tempranas como por el impacto que pueda tener en la sensibilidad de los más jóvenes la exposición a productos literarios y culturales diversos, contribuyendo a la cimentación de la competencia cultural. Por ello, tras la revisión teórica realizamos una propuesta de lecturas destinadas a niños de Educación Primaria que se encuentran aprendiendo inglés como segunda lengua.

\section{Planteamiento teórico-descriptivo}

Desde la geminación en los años 70 del enfoque comunicativo, basado en la "competencia comunicativa" (Hymes, 1972), su aplicación al ámbito de la enseñanza de lenguas extranjeras ha sido intensiva. Se persigue, como indica su nombre, la comunicación. Sin embargo, más allá de la sobreutilización del término (Lasagabaster, 2000) y de las diferentes controversias sobre este enfoque, como la falta de concreción sobre la formación del profesorado, su utilidad en diferentes niveles, el desarrollo de materiales y la falta de consideración a determinados aspectos formales del lenguaje (Richards y Rodgers, 2014), en el ámbito de la literatura el uso de documentos reales de lengua ha relegado su presencia a testimonios mínimos. Los documentos auténticos utilizados en el aula son normalmente cartas, mapas, artículos de periódicos, anuncios, etc., recursos que desarrollan la función referencial del lenguaje, dejando de lado la función expresiva (Donnerstag, 1996). Por otro lado, la competencia comunicativa forma parte del diseño curricular de nuestros sistemas de enseñanza, con lo cual dentro de esta coherencia es necesario retomar la literatura en el aula de L2.

Vemos, pues, que "los textos literarios deben ser incluidos en la enseñanza de lenguas extranjeras siempre que la competencia comunicativa se comprenda como algo más que capacidad de reproducción" (Hunfeld, en Olsbu y Salkjelsvik, 2008, 869). 
En la misma línea Maley (1999) destaca la trivialidad de los materiales de enseñanza que aportan una visión de la vida simple y sin controversias o problemáticas. Sell añade, además:

En los libros de texto no aparecen temas como las relaciones humanas, el sexo, las distintas orientaciones sexuales, las drogas, el alcohol, el racismo, la soledad, el miedo, el bullying, la violencia, el hacerse mayor, la muerte, etc. La literatura puede cubrir este espacio en blanco en la enseñanza, introduciendo estos temas a través de un material que es auténtico y tiene la potencialidad de hacer que los aprendientes se involucren afectivamente (Sell, 2005, 92).

Sin embargo, la situación que nos encontramos es que el Marco Común Europeo de Referencia para las Lenguas (MCERL) prácticamente no contempla la literatura en este proceso, tampoco figura la competencia literaria ni el uso de textos literarios para desarrollar ninguna otra competencia (Olsbu y Salkjelsvik, 2008), en parte por la dificultad intrínseca de medir en términos evaluables aquello que el alumno es capaz de hacer.

Además, en la actualidad, la oralidad del lenguaje ha desbancado por completo a la escritura y la literatura parece estar relegada tanto de los métodos como de los materiales (Pastor, 2004), como respuesta a métodos más tradicionales que contaban exclusivamente con las destrezas escritas como herramienta de aprendizaje.

Es decir, la ausencia de literatura en el aula de segundas lenguas supone una merma significativa tanto de temáticas como de exposición a las posibilidades que un determinado idioma ofrece. Además, si la motivación es un factor determinante en el aprendizaje y la adquisición, debemos ofrecer a los estudiantes textos exigentes, interesantes y estimulantes que hagan que se involucren a un nivel más profundo, sintiendo que están usando la lengua con objetivos reales (Daskalovska, y Dimova, 2012). La literatura es una de las formas de comunicación más desarrolladas y exigentes y, por lo tanto, es un elemento ineludible para un enfoque que se define a sí mismo como comunicativo (Lasagabaster, 2000).

Hay que reconocer que aunque recientemente se ha generado cierto interés por incluir la literatura en la clase de segundas lenguas, ya que como señala Langer (1995) esta juega un papel muy importante en nuestras vidas. Apunta Amer (2012) además que, aunque no nos demos cuenta tiene una dimensión humanizadora al mismo tiempo que supone una provocación intelectual por proporcionar diferentes puntos de vista para examinar nuestros propios pensamientos, aún su uso no es extensivo ${ }^{3}$.

Uno de las aportaciones más significativas que viene de la mano de la literatura y que hoy en día cada vez es más demandada por el mundo globalizado en el que vivimos es la multiculturalidad o la interculturalidad, hasta el punto de ser una de las características que define nuestra sociedad occidental (Sell, 2005). Así, el argumento por excelencia para defender la presencia de la literatura en la clase de segundas lenguas es su inherente componente cultural. La literatura puede fomentar la con-

Varios autores han señalado ya la importancia de la literatura en el proceso de enseñanza-aprendizaje de segundas lenguas, razón por la cual no entraremos en detalle. Remitimos a los trabajos de Brumfit, 1991; Lasagabaster, 1999; Lazar, 1993; Nunan, 1998; Gilroy y Parkinson, 1997; Byram, Morgan et al., 1994; Parkinson y Thomas, 2000; Maley y Moudling, 1985; Duff y Maley, 1990. 
ciencia cultural, étnica y religiosa, y la valoración de la diversidad y la sensibilidad hacia otras perspectivas y visiones del mundo (Stotsky, 1994; Naidoo, 1999).

La literatura se convierte, pues, en la herramienta más eficaz para desarrollar una competencia cultural afectiva que facilite a los alumnos su relación positiva con la cultura de la lengua meta dentro de los parámetros de la misma (Sell, 2002a, 266), favoreciendo así el desarrollo de una competencia cultural real que posibilite la socialización y la efectividad en la lengua meta.

Esta situación ya demostrada nos obliga a plantearnos qué cultura o qué culturas tienen que llegar a nuestra aula. La diversidad es esencial, se hace imperiosa la presencia de la cultura colonial y de la cultura de la globalización a través del inglés como lengua global o lingua franca. Es necesario, además, reconocer que no existe la pureza lingüística y que el objetivo de los aprendientes de lengua inglesa no es adquirir una competencia nativa sino dominar precisamente el inglés como lingua franca con sus innumerables variaciones regionales en todas las unidades del idioma. (Graddol, 1999 y 2001, y Seidlhofer, 2001). Por lo tanto, siguiendo los postulados de Sell (2005) la literatura es una valiosa herramienta para potenciar la conciencia y la competencia cultural, especialmente si vamos más allá de lo ofrecido por las literaturas británicas y americanas. Es decir, la presencia de una literatura que refleja las variedades lingüísticas del inglés sirve también como modelo multicultural, como señalamos más abajo.

De hecho si atendemos a los cinco componentes que constituyen la competencia comunicativa en el MCERL, lingüístico, pragmático-discursivo, estratégico, personal y socio-cultural, todos se desarrollan a través de la literatura y el último, especialmente, en el tipo de propuesta que aquí queremos elaborar. Consideramos, en la línea de Crystal (1997) que el nuevo rol del inglés como lingua franca juega un papel fundamental en la diversidad cultural de nuestra sociedad que debemos reflejar en el proceso de enseñanza-aprendizaje del inglés que no se ha de obviar.

Así las cosas, en el ámbito de la enseñanza del inglés lo que acabamos de plantear tiene una especial importancia por su carácter global. Tal y como afirma Crystal $(2003,10)$, "el inglés es la lengua en la que nunca se pone el sol" por lo que es necesario hablar de Englishes y no de English. La expansión del inglés como lengua colonial y luego como lengua oficial o semioficial hasta contar con presencia en todos los continentes, así como la expansión sociocultural del mismo que ha penetrado profundamente en la política internacional, economía, negocios, entretenimiento, medios de comunicación, educación, etc. lo han llevado a convertirse en una lingua franca ante las necesidades de un mundo globalizado (Crystal, 1997).

Esta generalización del uso del inglés a nivel internacional ha supuesto la implantación masiva de prácticas educativas destinadas a dotar a la población de las herramientas lingüísticas necesarias para dominar la lingua franca. Ante lo que se plantea la problemática de los diferentes modelos de lengua inglesa existentes que constituyen un fenómeno muy complejo, tanto desde una perspectiva pedagógica como sociolingüística, cultural y económica. Así, existen controversias entre quienes reclaman un modelo estandarizado y monocéntrico y quienes abogan por un modelo pluricéntrico e interculturalmente significativo (Martín, 2010).

Jenkins $(2006,161)$ se sitúa en contra de la enseñanza de un inglés idéntico para todos. Afirma que una comunicación global demanda un repertorio lingüístico amplio y adecuado que contemple la noción de Englishes. El impulso de la conciencia del inglés como una lengua global permite además el desarrollo del modelo pluricén- 
trico, el cual permite a los estudiantes y hablantes reflejar su propia realidad sociolingüística y no aquella del hablante nativo que les resulta ajena y distante (Jenkins, 2006, 174). En la misma línea se sitúa Seidelhofer (2005) cuando afirma que hoy en día es difícil justificar la idea de un inglés estandarizado dado nuestro contexto.

Por otro lado, como señala Martín $(2010,36)$, la desconexión entre la investigación y la práctica docente en el ámbito del inglés ha llevado a una desorientación del profesorado sobre qué variantes o variante del idioma hay que impartir y cómo llevar al aula esta riqueza lingüística de tal forma que el modelo centralizado, inglés estandarizado y monocéntrico, sigue prevaleciendo.

En este panorama lingüístico, la literatura juega un papel fundamental. Así, a pesar de la tradicional asociación entre el sintagma literatura en lengua inglesa con la literatura británica y/o estadounidense, poco a poco se van integrando al corpus obras de Australia, Canadá, Nueva Zelanda, etc. y también, obras de contextos en los que el inglés convive con otras realidades lingüísticas como Nigeria, Kenia, India, Filipinas, El Caribe, Malasia, Singapur, etc. De hecho, en los últimos años obras de calidad de estos contextos han ganado prestigiosos premios internacionales (Carter y Long, 1991, 156). Escritores de todo el mundo, desde el Pacífico, Asia, África y las Indias hasta los núcleos tradicionales de Gran Bretaña y Estados Unidos, utilizan la lengua inglesa como medio de expresión literaria. La consecuencia de esto es que se ha producido una verdadera explosión en la literatura en inglés, que ha pasado a convertirse en literatura multicultural (Dasenbrock, 1987).

Así, es importante apreciar el valor de estos textos literarios multiculturales no sólo por la presencia de esta multiculturalidad dentro de su temática y argumento, sino por su enorme potencialidad a la hora de llegar a lectores de inglés como lengua global e inteligible, a pesar de las polémicas existentes en cuanto a la comprensión o no de textos tan dispares culturalmente (Dasenbrock, 1987).

$\mathrm{Si}$, como veíamos anteriormente, la presencia de literatura en el aula de segundas lenguas forma parte esencial del trabajo de la competencia comunicativa, es aún más importante en edades tempranas, ya que la enseñanza del inglés se está adelantando cada vez más en los planes educativos. Ghosn $(2002,172)$ establece al respecto que la literatura infantil es un medio motivador para adquirir segundas lenguas, al mismo tiempo que es positiva para el desarrollo de habilidades cognitivas necesarias de la competencia comunicativa, a pesar de lo cual, ni en Educación Primaria ni en Secundaria los profesores conocen el potencial de la literatura infantil en la enseñanza de segundas lenguas (Bland y Lütge, 2013).

\section{La literatura infantil en el aula de segundas lenguas. Situación}

La literatura infantil se constituye, pues, como un recurso muy válido para desarrollar, también, la competencia multicultural en el alumnado, haciendo necesario el poner a su alcance literatura diversa y culturalmente rica. Siguiendo a Naidoo (1999) podemos establecer que es necesario promover en el aula la multiculturalidad, la crítica y la creatividad más allá de facilitar el acceso a una literatura global. Así, la literatura se convierte en un recurso para compartir, disfrutar y reflexionar al mismo tiempo que es útil por sus dimensionas morales, sociales y culturales además de suponer un excelente punto de partida para explorar nuestra identidad y la de los demás. 
Vemos, pues, que el valor de la literatura como herramienta para fomentar la conciencia multicultural y la competencia comunicativa es innegable, ya que refleja aspectos de culturas diferentes a la propia: valores, creencias, formas de pensar, modos de vida... La introducción en las aulas de literatura multicultural de calidad resulta beneficiosa para apreciar la forma de ser de otros grupos, eliminar barreras etnocéntricas y desarrollar perspectivismo en la valoración de la diversidad. En este sentido, suscribimos las palabras de Dowd (1992, 220): "De leer, escuchar y utilizar materiales culturalmente diversos, los niños y jóvenes aprenden a ver más allá de las diferencias de color, etnia o cultura, interiorizando que todos los seres humanos experimentan los mismos sentimientos universales de amor, tristeza, valor y bondad".

Además, en línea con lo que plantea Lütge (2012) la literatura ofrece un mundo imaginativo y fantasioso que también favorece la conexión con la competencia comunicativa intercultural. Así, la literatura infantil puede jugar un papel muy importante en el desarrollo de una conciencia intercultural en el alumnado desde edades tempranas, algo imprescindible en la sociedad actual (Bland y Lütge, 2013).

El desarrollo de la comprensión de "el otro", así como la competencia comunicativa lingüística en conjunto es el gran objetivo de la enseñanza-aprendizaje de segundas lenguas para lo cual los textos literarios auténticos suponen un recurso incomparable siguiendo los principios de la "competencia comunicativa intercultural" (Byram, 1997) atendiendo a las necesidades del "hablante intercultural" (Kramsch, 1993).

La utilización de la narrativa, género más presente en las aproximaciones más tradicionales de aula de segundas lenguas, tiene también su sentido hoy en día. La novela infantil y juvenil presenta un valioso potencial para fomentar la empatía y la comprensión en los lectores, además de su componente motivacional. La narrativa, con narrador omnisciente o en primera persona, muestra directamente las ideas, pensamientos y sentimientos de los protagonistas. Siguiendo a Reichl (Bland y Lütge, $2013,110)$ y sus fases de aprendizaje en los textos narrativos podemos ver cómo resulta especialmente idóneo para entender la identidad particular de un texto a través de la implicación emocional e intelectual, las diferentes voces y subjetividades de la historia, así como las sensaciones de duda y de cuestionamiento que se detectan al ver otros patrones de pensamiento y otros esquemas culturales.

Los textos seleccionados para promover este aprendizaje multicutural a través del inglés suelen ser fáciles, de forma que permitan la lectura sin demasiadas interrupciones por dificultades lingüísticas. Sin embargo, cierta dificultad en los textos, especialmente respecto a las motivaciones e identidades de los personajes da lugar a situaciones de aprendizaje valiosas de forma que una novela infantil o juvenil con temas culturales en vez de ser considerada como una barrera para el lector puede ser considerada como una oportunidad de aprendizaje significativo sobre otras culturas e identidades (Bland y Lütge, 2013).

Dentro de la riqueza literaria que ofrecen lo diferentes géneros creemos que una de las herramientas más eficaces para acercar la literatura al aula de segundas lenguas, especialmente en edades tempranas, la constituye el híbrido genérico del álbum ilustrado. Sus peculiaridades a caballo entre el producto artístico plástico y el literario facilitan una interacción que va más allá de lo lingüístico. Dada, pues, la alfabetización visual del alumnado actual creemos que el álbum ilustrado es una herramienta de primer orden en la enseñanza de segundas lenguas y, en concreto, del inglés, como ya han señalado entre otros, Kress y Van Leeuwen (2006), Domínguez Pérez (2015), Martínez Mateo (2014), Dotras Bravo (2013) y Mier y Santamaría (2016). 


\section{Propuesta de mejora: dos lecturas}

Ante la situación que acabamos de relatar hacemos dos propuestas literarias que pueden llevarse a cabo en el contexto de Educación Primaria. Nuestra formulación se basa en dos textos concretos, Diary of a Wombat de French y Whatley, álbum ilustrado, y Journey to Jo 'burg, que es una novela infantil de la escritora sudafricana Beverley Naidoo, pero puede ser aplicada a muchos otros, tanto dentro del género álbum ilustrado como del género narrativo ${ }^{4}$.

El primero es un álbum ilustrado publicado en 2002 galardonado con diversos premios como el American Library Association Notable Children's Book o el Canberra's Own Outstanding List Award for Best Picture Book, entre otros. Su estructura en forma de diario, así como el uso de frases cortas incluso de una sola palabra y la calidad de las ilustraciones hace que sea muy apropiado para niños aprendientes de inglés. Está protagonizado por un wombat, un marsupial endémico de Australia y Tasmania con apariencia de oso pequeño de patas muy cortas, lo que facilita la asociación por parte de los niños con un nuevo contexto.

Dada la naturaleza del mismo creemos que las actividades de comprensión deben estar basadas en una metodología constructivista. Por ello, se comenzará la lectura a través de una actividad de calentamiento que sea una lluvia de ideas generada a raíz de la visualización de la portada del álbum en la pantalla. El profesor asumirá el papel del mediador y facilitador en el debate promoviendo siempre el uso de la L2. Para facilitar este trabajo puede guiar el debate a través de este esquema que refleja el léxico y los conceptos clave:

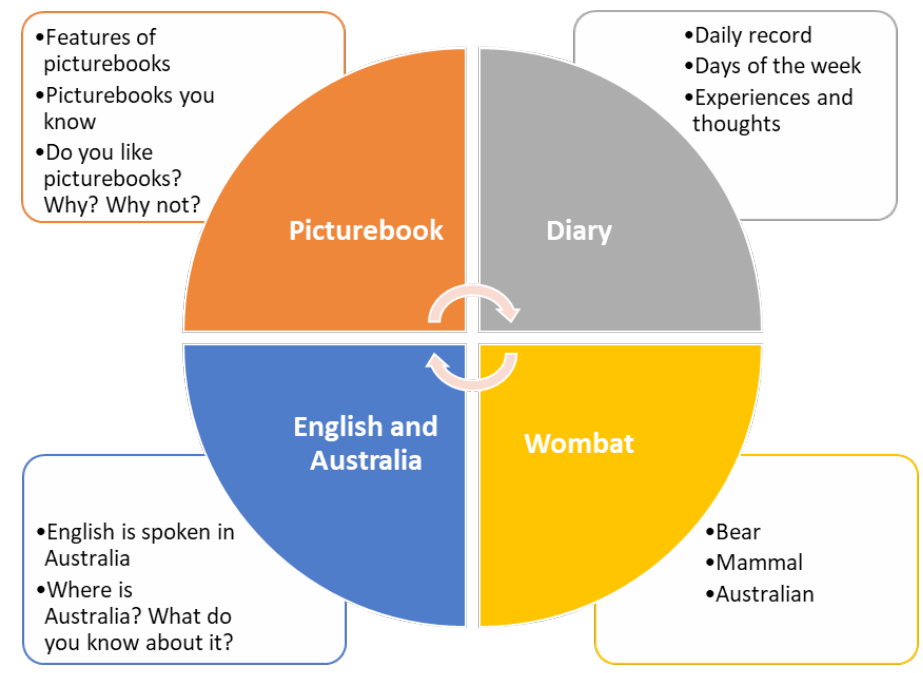

Figura 1. Léxico y conceptos clave.

$4 \quad$ La selección de textos aquí propuesta se ha hecho siguiendo las pautas establecidas por Beilke (1986), Harada (1995), Harris (1991), Pang, Colvin, Tran y Barba (1992) y Lu, M. Y. (1998), que podemos resumir en los siguientes rasgos: descripciones positivas de personajes evitando estereotipos y con conductas auténticas y realistas, ilustraciones auténticas que mejoren la calidad del material, diversidad temática, calidad literaria, precisión histórica, reflexividad sobre los valores culturales de los personajes, tramas que apoyen la concepción positiva de la diversidad cultural. 
Posteriormente se realizará una lectura colectiva a través de la proyección del álbum entero en la pantalla. A continuación se puede profundizar en el conocimiento de los wombats a través de preguntas y de la realización de dibujos. Para reflexionar sobre la naturaleza del género álbum ilustrado podemos tomar como ejemplo la escena en la que el wombat lucha contra una "flat hairy creature who is invadig his territory" (un felpudo), aprovechando este gracioso malentendido para que dibujen y piensen en otros objetos que el protagonista pueda confundir con alguna criatura peligrosa, completando el álbum ilustrado con sus aportaciones. Podemos también aprovechar el formato de diario del texto para trabajar con ellos esta rutina, en la que se puede profundizar sobre la diferencia horaria y las consecuencias de la rotación de la Tierra en el horario mundial.

Nuestra segunda lectura propuesta, Journey to Jo'burg es una novela infantil de la escritora sudafricana Beverley Naidoo que se contextualiza en la etapa del apartheid en Sudáfrica. Su argumento es el siguiente: dos niños negros de 13 y 9 años, cuya hermana pequeña está enferma, van a buscar a su madre, quien trabaja en Johannesburgo como criada. Este viaje sirve para ilustrar la realidad el apartheid en Sudáfrica: ruptura de familias, ley de pases, racismo, desigualdad, etc.

A pesar de la complejidad que presenta esta obra, ya que requiere cierto dominio del inglés, así como del contexto histórico de Sudáfrica, creemos que utilizarla puede ser muy enriquecedor por el desarrollo de competencias básicas generales que conlleva: sociales, cívicas, culturales, etc. Además la historia permite un fácil proceso de empatía con los protagonistas y que se desarrolla en un contexto histórico muy complejo e interesante. Así, confluyen en un mismo recurso el input lingüístico, el componente del inglés como lengua multicultural y la compleja y atractiva historia de Sudáfrica, resultando en una alta motivación para el alumnado y en multitud de posibilidades didácticas transversales.

Para esta lectura en concreto creemos que es especialmente apropiado el formato del Club de Lectura. El club de lectura escolar fomenta el gusto por la lectura y permite el desarrollo de actividades comunicativas realmente significativas (Arana-Palacios y Galindo-Lizaldre, 2009; Jiménez-Fernández y Cremades-García, 2013). Gritter (2011) expone que la interacción grupal, la participación y la escucha promueven la implicación personal de los alumnos, aumentando así la motivación y estableciendo vínculos significativos entre la literatura y la vida. Atwell (2007) y Cassany (2013) también argumentan que este tipo de iniciativas también promueven el desarrollo de la dimensión crítica de la lengua (Álvarez-Álvarez y Pascual-Díez, 2014). Además, dentro de la temática que nos ocupa el formato parece especialmente indicado para la educación en valores (Álvarez y Gutiérrez, 2013), además de la lingüística (Cerrillo y García Padrino, 1996), literaria (Lage, 2006; Lockwood, 2008), educativa (Atwell, 2007; Jurado, 2008) cívica y social (Sánchez y Yubero, 2004).

Para llevar a cabo esta propuesta será necesario facilitar previamente las herramientas léxicas necesarias por la complejidad que se plantea en L2. Así como también puede ser necesaria cierta familiarización con la realidad socio-histórica de Sudáfrica. Las sesiones se irán organizando con los 15 capítulos de la obra, de forma que se vayan poniendo en común y se vayan comprendiendo paulatinamente. 


\section{Conclusiones}

A pesar de su brevedad, creemos que las propuestas que aquí lanzamos sirven para perfilar el tipo de actividades que se pueden llevar a cabo en al aula, más allá de la siempre útil lectura placentera, con literaturas procedentes de otras culturas y que reflejan otras variedades del inglés de las más manejadas mayoritariamente. De esta forma, se contribuye al fomento de la competencia cultural en nuestros alumnos y de la competencia literaria.

Como hemos demostrado en la primera parte del trabajo, la necesidad de ampliar el canon de lecturas en segundas lenguas y adaptarlas a las circunstancias sociales y lingüísticas que rodean a nuestros discentes es uno de los retos a los que se enfrenta el profesorado hoy en día, de ahí que presentemos esta propuesta sobre literatura infantil no adaptada cuyo componente cultural es parte de su definición. La literatura es una herramienta significativa que da pie tanto al conocimiento como a la reflexión del inglés y de la historia de la lengua en nuestro planeta y debemos aprovecharnos de ello.

Tanto la diversidad genérica en la literatura presentada como la diversidad cultural supondrán una fuente rica para que el alumno de Educación Primaria desarrolle su competencia comunicativa en nuestro mundo, de forma que vaya siempre de la mano de la competencia cultural y, también, aunque en desigual medida, de la competencia literaria. Creemos que las propuestas como las que aquí se perfilan contribuyen a un desarrollo holístico de las competencias básicas dentro de un plan integrado.

\section{Referencias bibliográficas}

Álvarez Álvarez, Carmen, y Raquel Gutiérrez Sebastián (2013): "Educar en valores a través de un club de lectura escolar: un estudio de caso / Educate in values through a school book club: a case study", Revista complutense de educación, 24.2, 303-319.

Álvarez Álvarez, Carmen, y Julián Pascual Díez (2014): “Aportaciones de un club de lectura escolar a la lectura por placer", El profesional de la información, 23.6, 625-631.

Amer, Aly Anwar (2012): Using Literature in Reading English as Second / Foreign Language, Tanta (Egipto), Tanta University.

Arana Palacios, Jesús, y Belén Galindo Lizaldre (2009): Leer y conversar: una introducción a los clubes de lectura, Gijón, Trea.

Atwell, Nancie (2007): The reading zone: How to help kids become skilled, passionate, habitual, critical readers, New York, Scholastic.

Beilke, Patricia F. (1986): Selecting materials for and about Hispanic and East Asian children and young people, Hamden (Conneticut), Library Professional Publications.

Bland, Janice, y Christiane Lütge (2013): Children's Literature in Second Language Education, Londres, Bloomsbury.

Brumfit, Christopher (1991): "Introduction: Teaching English to children”, en C. Brumfit, J. Moon y R. Tongue (Eds.), Teaching English to children, Londres, Collins.

Byram, Michael (1997): Teaching and Assessing Intercultural Communicative Competence, Frankfurt, Multilingual Matters Ltd.

Byram, Michael, Carol Morgan et al. (1994): Teaching and learning language and culture, Philadelphia, Clevendon. 
Carter, Ronald. A., y Michael N. Long (1991): Teaching literature, Londres, Addison-Wesley Longman Limited.

Cassany, Daniel (2002): "Mi taller de escritura", Textos, 30, 21-31.

Cassany, Daniel (2013): "Prácticas letradas vernáculas: lo que leen y escriben hoy los jóvenes", en Unplugged, la palabra como nueva tecnología, Santander, Universidad de Cantabria, 2013, 1-18.

Crystal, David (1997): English as a global language, Cambridge, Cambridge University Press.

Crystal, David (2003): English as a global language, segunda edición, Cambridge, Cambridge University Press.

Cerrillo Torremocha, Pedro, y Jaime García Padrino (1996): Hábitos lectores y animación a la lectura, Cuenca, Universidad de Castilla-La Mancha.

Dasenbrock, Reed W. (1987): "Intelligibility and meaningfulness in multicultural literature in English", Publications of the Modern Language Association of America, 102.1, 10-19.

Daskalovska, Nina, y Violeta Dimova (2012): "Why should literature be used in the language classroom?", Procedia-Social and Behavioral Sciences, 46, 1182-1186.

Domínguez Pérez, Mónica (2015): Los álbumes ilustrados para aprender español, Ocaña, Lastura.

Dotras Bravo, Alexia (2013): "El álbum ilustrado en la enseñanza de lenguas extranjeras: literatura y lengua", en S. Álvarez Ledo, M. Ferreira Boo y M. Neira Rodríguez (Eds.), De la literatura infantil a la promoción de la lectura, Madrid, Fundación Universitaria San Pablo, 139-147.

Donnerstag, Jiirgen (1996): "Improving the Expressive Competence of Foreign-Language Learners through Literary Reading”, en L. Bredella y W. Delanoy (Eds.), Challenges of Literary Texts in the Foreign Language Classroom, Tuebingen, Gunther Narr.

Dowd, Frances S. (1992): “Evaluating children's books portraying Native American and Asian cultures", Childhood Education, 68.4, 219-224.

French, Jackie, y Bruce Whatley (2006): Diary of a Wombat, Nueva York, Clarion Books.

Ghosn, Irma K. (2002): "Four good reasons to use literature in primary school ELT", ELT Journal, 14, 172-179.

Gilroy, Marie, y Brian Parkinson (1997): “Teaching literature in a foreign language", Language Teaching, 29.4, 213-225.

Graddol, David (1999): The Future of English, Londres, British Council.

Graddol, David (2001): "English in the future", Burns y C. Coffin (Eds.), Analysing English in a Global Context, Londres, Routledge, 26-37.

Gritter, Kristine (2011): "Promoting Lively Literature Discussion", The Reading Teacher, 64, 445-449.

Harada, Violet (1995): "Issues of ethnicity, authenticity, and quality in Asian American picture books, 1983-1993", Journal of Youth Studies, 8.2, 135-149.

Harris, Violet J. (1991): “Multicultural Curriculum: African American children's literature", Young Children, 46.2, 37-44.

Hymes, Dell H. (1972): “On Communicative Competence”, en J. B. Pride y J. Holmes (Eds.), Sociolinguistics. Selected Readings, Mamondsworth, Penguin, 269-293.

Jiménez Fernández, Concepción M., y Raúl Cremades García (2013): Bibliotecas escolares, Barcelona, UOC.

Jurado, Fabio (2008): "La formación de lectores críticos desde el aula", Revista Iberoamericana de Educación, 46, 89-105. 
Kramsch, Claire (2003): Context and Culture in Language Teaching, Oxford, Oxford University Press.

Kress, Gunther, y T. Van Leeuwen (2006): Reading Images. The Grammar of Visual Design, Londres, Routledge.

Lage, Juan. J. (2006): Animar a leer desde la biblioteca, Madrid, CCS.

Langer, J. A. (1995): Envisioning Literature: Literary Undestanding and Literature Instruction, Nueva York, Teachers College.

Lasagabaster, David (1999): "Literary awareness in the foreign language classroom", Cultura y Educación, 14-15, 5-17.

Lasagabaster, D. (2000): "El papel de la literatura en la clase de lengua extranjera", Cultura y Educación, 12.3, 67-86

Lazar, Gillian (1993): Literature and language teaching: a guide for teachers and trainers, Cambridge, Cambridge University Press.

Lockwood, Michael (2008): Promoting reading for pleasure in the Primary School, Londres, SAGE.

Lu, Mei-Yu (1998): Multicultural children's literature in the elementary classroom, Indiana, ERIC Clearinghouse on Reading, English, and Communication.

Lütge, Christiane (2012): "Developing 'Literary Literacy'? Towards a Progression of Literary Learning”, en M. Eisenmann y T. Summer (Eds.), Basic Issues in EFL teaching and Learning, Heidelberg, Winter, 191-202.

Maley, Alan (1999): Short and Sweet: Short texts and how to use them, Harmondsworth, Penguin.

Maley, Alan, y Alan Duff (1990): Literature, Oxford, Oxford University Press.

Maley, Alan, y Sandra Moulding (1985): Poem into Poem, Cambridge, Cambridge University Press.

Martín Gutiérrez, Félix (2010): “A vueltas con la globalización del inglés: expectativas y paradojas”, Historia y Comunicación Social, 15, 29-49.

Martínez Mateo, Roberto (2014): "The story of Ferndinand: de Nueva York a Salamanca", Ocnos, 12, 25-56.

Mier Pérez, Laura, y Susana Santamaría Fernández (2016): "El álbum ilustrado en Primaria: un recurso para la adquisición de L2", Lenguaje y textos, 14, 31-38.

Naidoo, Beverley (1999): "Heritage or Multiculturalism”, en R. Stones (Ed.) A Multicultural Guide to Children's Books 0-16, Londres, Books For Keeps / Reading and Language Information Centre.

Naidoo, Beverly (2002): Journey to Jo 'burg, Londres, Harper Collins Children's Book.

Nunan, David (1988): Syllabus Design, Oxford, Oxford University Press.

Nunan, David (2003): “The Impact of English as a Global Language on Educational Policies and Practices in the Asia` Pacific Region”, TESOL Quarterly, 37.4, 589-613.

Olsbu, Inger, y Kari Soriano Salkjelsvik (2008) "Objetos perdidos: La literatura en la clase de E/LE. Europa y Noruega”, Hispania, 91.4, 865-876.

Pang, Valerie Olga, Carolyn Colvin, MyLoung Tran y Robertta H. Barba (1992): "Beyond chopsticks and dragons: Selecting Asian-American literature for children", The Reading Teacher, 46.3, 216-224.

Parkinson, Brian, y Helen Reid Thomas (2000): Teaching Literature in a Second Language, Edinburgh, Edinburgh University Press.

Pastor Cesteros, Susana (2004): Aprendizaje de segundas lenguas. Lingüística aplicada a la enseñanza de idiomas, Alicante, Universidad de Alicante. 
Richards, Jack C., y Theodore S. Rodgers (2014): Approaches and methods in language teaching, Cambridge, Cambridge University Press.

Sánchez García, Sandra, y Santiago Yubero Jiménez (2004). "La lectura como elemento integrador de valores para la adaptación social”, en Elisa Larrañaga Rubio (coord.), Miradas a lo social. Procesos y problemas sobre los que actúa el trabajo social, Cuenca, Universidad de Castilla-La Mancha, 197-206.

Seidlhofer, Barbara (2001): "Closing a conceptual gap: the case for a description of English as a lingua franca", International Journal of Applied Linguistics, 11, 133-158.

Sell, Jonathan P. A. (2005): "Why teach literature in the English classroom?", Encuentro. Journal of Research and Innovation in the Language Classroom, 15, 86-93.

Stostky, Sandra (1994): “Academic Guidelines for Selecting Multiethnic and Multicultural Literature", The English Journal, 83.2, 27-34. 